\title{
Down-regulation of CXCR2 on Neutrophils in Severe Sepsis Is Mediated by Inducible Nitric Oxide Synthase-derived Nitric Oxide
}

\author{
Fabrício Rios-Santos ${ }^{1,2 *}$, José C. Alves-Filho ${ }^{1 *}$, Fabrício Oliveira Souto ${ }^{2}$, Fernando Spiller ${ }^{1}$, Andressa Freitas ${ }^{1}$, \\ Celina Monteiro C. Lotufo', Milena Botelho Pereira Soares ${ }^{3}$, Ricardo Ribeiro dos Santos ${ }^{3}$, \\ Mauro M. Teixeira ${ }^{4}$, and Fernando de Queiroz Cunha ${ }^{1 *}$ \\ 'Department of Pharmacology, Faculty of Medicine of Ribeirão Preto, University of São Paulo, São Paulo, Brazil; ${ }^{2}$ Department of Health, \\ University of Santa Cruz, Bahia, Brazil; ${ }^{3}$ Laboratory of Tissue Engineering and Immunopharmacology, Gonçalo Moniz Research Center/ \\ FIOCRUZ, Bahia, Brazil; and ${ }^{4}$ Laboratory of Immunopharmacology, Department of Biochemistry and Immunology, Institute of Biological \\ Science, Federal University of Minas Gerais, Belo Horizonte, Brazil
}

\begin{abstract}
Rationale: The failure of neutrophils to migrate to an infection focus during severe sepsis is an important determinant of the inability of a host to deal with an infectious insult. Our laboratory has shown that inducible nitric oxide synthase (iNOS) induction and NO production contribute to the failure of neutrophils to migrate in the context of sepsis.

Objectives and Methods: We investigated whether CXCR2 expression contributed to the failure of neutrophils to migrate during severe sepsis and the role of $\mathrm{NO}$ in modulating CXCR2 expression on neutrophils in mice subjected to nonsevere (NS) or severe (S) cecal ligation and puncture (CLP).

Results: Neutrophil migration to the infection focus was deficient in S-CLP mice, a phenomenon prevented by pharmacologic (aminoguanidine, L-canavanine) or genetic (iNOS gene deletion) inhibition of iNOS. The expression of CXCR2 on neutrophils from S-CLP mice was significantly reduced when compared with neutrophils from NS-CLP or sham-operated mice. CXCR2 expression was reestablished by pharmacologic and genetic inhibition of iNOS. Immunofluorescence and confocal analysis revealed that iNOS blockade reduced neutrophil CXCR2 internalization. Adhesion and emigration of neutrophils in macrophage inflammatory protein-2-stimulated mesentery microcirculation were reduced in S-CLP mice, compared with NS-CLP mice, and reestablished by pretreatment with aminoguanidine or L-canavanine. The NO donor S-nitroso-N-acetyl-D,Lpenicillamine inhibited CXCL8-induced human neutrophil chemotaxis and CXCR2 expression on human and murine neutrophils. Conclusion: These results highlight evidences that the failure of neutrophils to migrate to an infection focus during severe sepsis is associated with excessive NO production and NO-dependent regulation of the expression of CXCR2 on the neutrophil surface.
\end{abstract}

Keywords: sepsis; neutrophil migration; CXCR2; nitric oxide

The migration of neutrophils to an infection focus constitutes the first line of defense against infection (1-3). The migrated

(Received in original form January 24, 2006; accepted in final form November 30, 2006)

* These investigators contributed equally to this study.

Supported by grants from the Fundação de Amparo à Pesquisa do Estado de São Paulo (FAPESP), Coordenação de Aperfeiçoamento de Pessoal de Nível Superior (CAPES), Conselho Nacional de Pesquisa e Desenvolvimento Tecnológico (CNPq), and Programa de Núcleos de Excelência (PRONEX)

Correspondence and requests for reprints should be addressed to Fernando Q. Cunha, Ph.D., Department of Pharmacology, Faculty of Medicine of Ribeirão Preto, University of São Paulo, Av. Bandeirantes 3900, Monte Alegre, 14049-900 Ribeirão Preto, SP, Brazil. E-mail: fdqcunha@fmrp.usp.br

This article has an online supplement, which is accessible from this issue's table of contents at www.atsjournals.org

Am J Respir Crit Care Med Vol 175. pp 490-497, 2007

Originally Published in Press as DOI: 10.1164/rccm.200601-1030C on November 30, 2006 Internet address: www.atsjournals.org

\section{AT A GLANCE COMMENTARY}

Scientific Knowledge on the Subject

Nitric oxide (NO) participates in the pathogenesis of sepsis and the chemokine receptor CXCR2 has been shown to be down-regulated on neutrophils during severe sepsis.

\section{What This Study Adds to the Field}

Failure of migration of neutrophils to an infection focus during severe sepsis is associated with excessive NO production and NO-dependent down-regulation of CXCR2 expression on the neutrophil surface.

neutrophils may control bacterial growth and, consequently, prevent bacterial dissemination and death. Failure of neutrophils to migrate to an infection focus is a key event responsible for the inability of a host to restrict the growth and dissemination of a pathogen $(4,5)$. Systemic released nitric oxide (NO) appears to be a major mediator of the impairment of neutrophil migration in sepsis after cecal ligation and puncture (CLP), Staphylococcus aureus injection and endotoxemia model (6-11). Consistently, in patients with sepsis or severe trauma, the observed inhibition of neutrophil chemotaxis was accompanied by increased serum concentrations of chemokines and NO metabolites $(12,13)$. Thus, although NO production may be relevant for the ability of a host to deal with an infectious insult $(14,15)$, overproduction of $\mathrm{NO}$ in the circulation prevents neutrophil migration and might be detrimental to the outcome of sepsis.

Neutrophil recruitment to sites of infection is heavily dependent on chemokines, mainly CXC chemokines containing the tripeptide Glu-Leu-Arg (CXC-ELR ${ }^{+}$chemokines) (16). Human peripheral blood neutrophils express two major subtypes of chemokines receptors, CXCR1 and CXCR2 $(17,18)$, whereas murine neutrophils express the CXCR2 receptor (19). Whereas degranulation and calcium flux in response to CXCL8 are mediated by both receptors, respiratory burst and chemotaxis are mediated mainly by CXCR1 (20) and CXCR2 (21), respectively. The importance of CXC-ELR ${ }^{+}$chemokines and their receptors for host defense against infection has been demonstrated in various models. Mice deficient in lungkine (CXCL15) had a decrease in neutrophil migration to the infection focus and an increase in mortality rate after Legionella pneumophila challenge (22). The pneumonia induced by Aspergillus fumigatus after CXCR2 blockade is indistinguishable from that obtained after neutrophil depletion and much more severe than in the presence 
of neutrophils (23). In addition, CXCR2 is an essential chemokine receptor for the innate response against infection due to inoculation with Pseudomonas aeruginosa (24) and Nocardia asteroides (25).

An important feature of seven-transmembrane domain $G$ protein-coupled receptors (GPCRs), including chemokine receptors, is the rapid internalization of the receptor from the cell surface into endosomal compartments on agonist stimulation (26). Both CXCR1 and CXCR2 are down-regulated on stimulation with CXCL8. After removal of the chemokine, CXCR1 is rapidly and completely reexpressed on the cell surface, whereas CXCR2 reexpression is slow and only partial $(21,27)$. In patients with severe sepsis or trauma, there was a reduction in the expression of CXCR2 on neutrophils, but not of CXCR1 (28-31). However, it is not known whether CXCR2 down-regulation plays a relevant role in the failure of neutrophils to migrate to an infectious focus and whether NO plays a role in CXCR2 down-regulation. In the present study, we demonstrated that CXCR2 plays a critical role in the migration of neutrophils elicited by CLP in mice. Moreover, we provide the first evidence to suggest that NO down-regulates CXCR2 expression on neutrophils and prevents CXCR2-dependent neutrophil recruitment to a site of infection during severe sepsis.

\section{METHODS}

Experimental details are provided in the online supplement.

\section{Animals}

C57BL/6 (8- to 12-week-old) male mice obtained from the animal facility of our institution (University of São Paulo, São Paulo, Brazil) and C57BL/6 inducible nitric oxide synthase-deficient $\left(\mathrm{iNOS}^{-/}\right)$mice purchased from Jackson Laboratory (Bar Harbor, ME) were housed under standard conditions and received water and food ad libitum. All experiments were conducted according to the guidelines laid down by the ethics committee of the School of Medicine of Ribeirão Preto, University of São Paulo.

\section{Sepsis Model}

The sepsis model used was CLP, as described (32). We have standardized the number of punctures in CLP required to display nonsevere (NS-CLP) and severe (S-CLP) effects in control mice. Briefly, mice were anesthetized and the cecum was exposed, ligated, and punctured twice with a 24-gauge needle (NS-CLP) or 14 times with a 21-gauge needle (S-CLP). Next, the cecum was repositioned in the abdomen, and the peritoneal wall was closed. Sham-operated animals underwent identical laparotomy but without cecal puncture. The survival rate was determined daily for 5 days after surgery.

\section{Pharmacologic Treatments}

Mice were treated subcutaneously with saline (vehicle) or with selective iNOS inhibitors, that is, aminoguanidine $(30 \mathrm{mg} / \mathrm{kg}$; Sigma, St. Louis, $\mathrm{MO}$ ) or L-canavanine (50 mg/kg; Sigma), $30 \mathrm{~min}$ before surgery. In another set of experiments, mice were pretreated with repertaxin (RTX, $30 \mathrm{mg} / \mathrm{kg}$, intravenous), a noncompetitive allosteric blocker of CXCR1 and CXCR2 (33), 30 min before surgery. RTX was a kind gift from R. Bertini (Dompé SpA, L’Aquila, Italy).

\section{Neutrophil Migration to Peritoneal Cavity}

Neutrophil migration was assessed 6 hours after CLP, as reported (8). The results are expressed as the number of neutrophils per cavity.

\section{Blood Bacterial Counts}

Bacterial counts were performed on blood samples collected 6 hours after CLP, as described (6).

\section{Neutrophil Isolation}

Mouse blood neutrophils were isolated by immunomagnetic negative selection, as previously described (34). Human blood neutrophils from healthy volunteers were isolated by Percoll density gradient centrifugation, as described (35).

\section{Flow Cytometry Analysis}

Mouse blood neutrophils were stained with phycoerythrin-conjugated anti-CXCR2 antibody (Santa Cruz Biotechnology, Santa Cruz, CA) or appropriate isotype controls. Human blood neutrophils were stained with rabbit anti-CXCR1 or anti-CXCR2 (Santa Cruz Biotechnology), and binding of these monoclonal antibodies was detected with fluorescein isothiocyanate-conjugated anti-rabbit IgG and compared with relevant isotype controls (Dako, Ely, UK). The cells were washed, fixed, and analyzed with a flow cytometer (BD FACSort; BD Biosciences, Mountain View, CA).

\section{Immunofluorescence Assay for CXCR2 Analysis}

Purified mouse neutrophils were affixed to glass slides and incubated with primary rabbit anti-CXCR2 IgG antibody or isotype controls (Santa Cruz Biotechnology). Next, secondary antibody (goat anti-rabbit $\mathrm{IgG}$ conjugated with green fluorescent Alexa-Fluor 488; Invitrogen Molecular Probes, Eugene, OR) was added. Images of marked cells were captured with an epifluorescence microscope (BX-50; Olympus, Tokyo, Japan).

\section{Intravital Microscopy of Mesenteric Microcirculation}

Leukocyte adhesion and migration were examined by the intravital microscopy technique, as described (7). To induce the leukocyteendothelium interaction, macrophage inflammatory protein (MIP)-2 $(20 \mathrm{ng} / \mathrm{ml})$ was applied directly to the mesenteric tissue, and cells in the recorded image were counted with a television camera incorporated onto a microscope (Carl Zeiss, Oberkochen, Germany).

\section{Chemotaxis Assays}

Purified human neutrophils $\left(10^{6}\right.$ cells $/ \mathrm{ml}$ in RPMI) were incubated at $37^{\circ} \mathrm{C}$ for 2 hours either in the absence (control) or in the presence of $S$-nitroso- $N$-acetyl-D,L-penicillamine (SNAP, $300 \mu \mathrm{M})$, citrulline $(30 \mu \mathrm{M})$, or CXCL8 $(30 \mathrm{ng} / \mathrm{ml})$, before chemotaxis to CXCL8 $(10 \mathrm{ng} / \mathrm{ml})$ in Boyden chamber assays, as previously described (13). To test the effect of NO on the inhibitory activity of CXCL8, neutrophils were incubated with aminoguanidine $(50 \mu \mathrm{M})$ at $37^{\circ} \mathrm{C}$ for $30 \mathrm{~min}$ before addition of the cytokine.

\section{Calcium Imaging Analysis}

Purified mouse neutrophils were incubated with Fluo-3-AM (Invitrogen Molecular Probes) and calcium mobilization in response to MIP-2 $(20 \mathrm{ng} / \mathrm{ml})$ was assayed by confocal microscopy (TCS SP5; Leica Microsystems USA, Bannockburn, IL).

\section{Statistical Analysis}

Data are reported as means \pm SEM of values obtained from two different experiments. The means between different treatments were compared by analysis of variance. If significance was determined, individual comparisons were subsequently tested by Bonferroni $t$ test for unpaired values. Bacterial counts were analyzed by Mann-Whitney U test. The survival rate was expressed as the percentage of live animals, and the Fisher exact test was used to determine differences in survival curves. $\mathrm{p}<0.05$ was considered significant. Data were analyzed with GraphPad Prism version 3.00 for Windows (GraphPad Software, San Diego, CA).

\section{RESULTS}

\section{Blockade of CXCR2 Inhibits Neutrophil Migration and} Enhances Mortality in Septic Peritonitis

Initial experiments were performed to determine the role of CXCR2 signaling in the outcome of sepsis. CXCR2 was inhibited with repertaxin (RTX, $30 \mathrm{mg} / \mathrm{kg}$, intravenous, $30 \mathrm{~min}$ before CLP), a noncompetitive allosteric blocker of CXCR1 and 

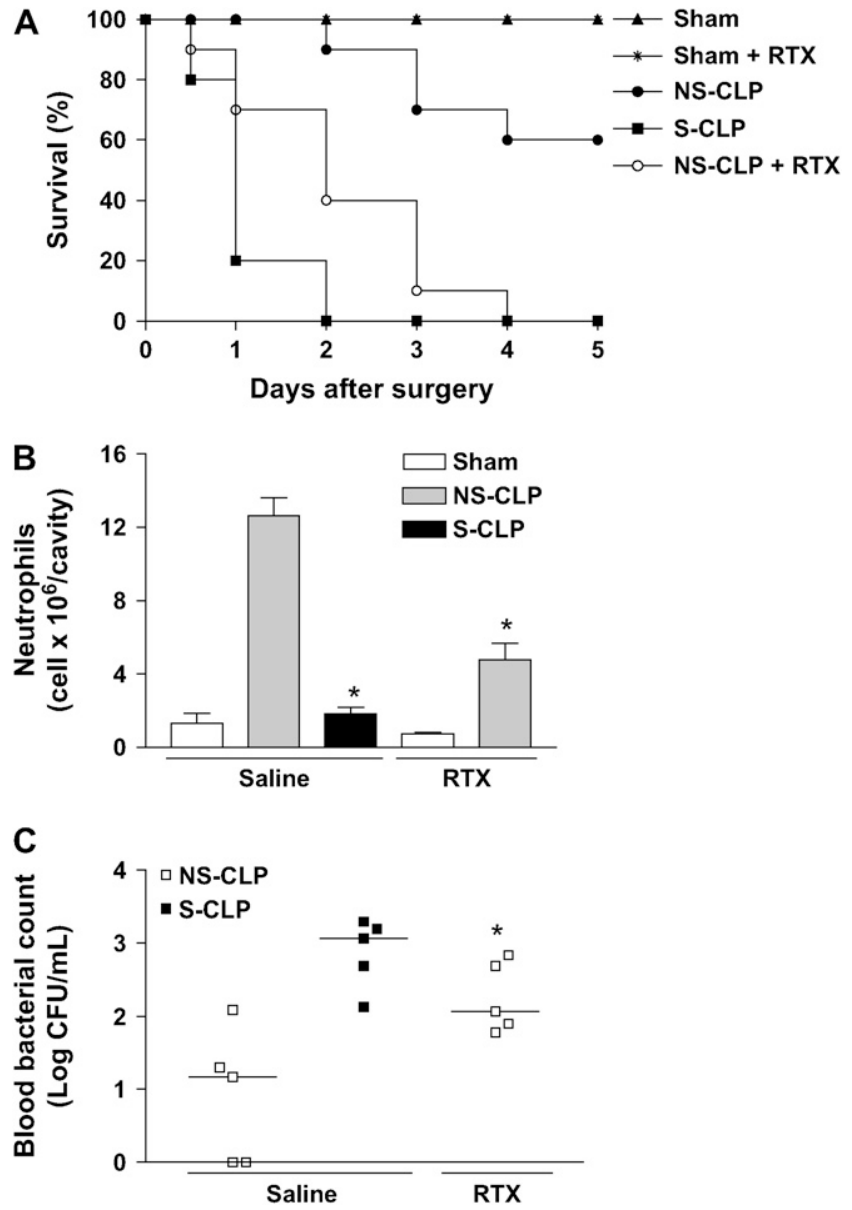

Figure 1. Blockade of CXCR2 inhibits neutrophil migration and enhances mortality in septic peritonitis. Shown are survival rates $(A)$, neutrophil migration into the peritoneal cavity $(B)$, and bacterial counts in blood $(C)$ of animals pretreated with saline (vehicle) or repertaxin (RTX, $30 \mathrm{mg} / \mathrm{kg}$, intravenous, $30 \mathrm{~min}$ before cecal ligation and puncture [CLP]) and subjected to sham surgery or nonsevere (NS)-CLP or of mice subjected to severe (S)-CLP that receive only vehicle. The survival rate of RTX-pretreated animals was significantly different from that of animals that received only vehicle after NS-CLP, with $p<0.05$, Fisher exact test $(n=12-15)$. The results of neutrophil migration are expressed as means and SEM of the number of neutrophils in the peritoneal cavity $(\mathrm{n}=10),{ }^{*} \mathrm{p}<0.05$ compared with NS-CLP group (analysis of variance, followed by the Bonferroni test). Results of bacterial counts are expressed as the log of colony-forming units (CFU) per milliliter of blood $(n=5)$, ${ }^{*} p<0.05$ compared with NS-CLP group (Mann-Whitney U test). No bacteria were detected in the sham and sham plus RTX groups.

CXCR2 (33). As shown in Figure 1A, the treatment of septic mice with repertaxin drastically decreased the survival of mice after nonsevere CLP (NS-CLP). Twenty-four hours after NSCLP, $100 \%$ of control mice were alive compared with $70 \%$ of repertaxin-treated mice $(n=10)$. The detrimental effects of inhibiting CXCR2 were most apparent on Day 4 after NS-CLP. At this time point, the survival rate among control mice was $60 \%$, whereas $100 \%$ mortality was observed in mice that received repertaxin. All sham-operated mice (sham and sham plus RTX) survived for 5 days after surgery, whereas $100 \%$ of animals subjected to S-CLP died on Day 2 after surgery $(\mathrm{n}=10)$.

Because neutrophil migration requires signaling via $\mathrm{CXCR} 2$, we next investigated whether blockade of CXCR2 affected neutrophil recruitment to the infectious focus and, consequently, the control of infection, as assessed by bacteremia. In agreement with our previous data $(8,9)$, the results in Figures $1 \mathrm{~B}$ and $1 \mathrm{C}$ show that animals subjected to NS-CLP presented marked neutrophil migration into the peritoneal cavity and low bacterial counts in blood 6 hours after surgery. However, mice subjected to S-CLP displayed impaired neutrophil migration compared with NS-CLP mice and failed to control the infection, as seen by the increased number of bacteria in blood (Figure 1C). Treatment of NS-CLP mice with repertaxin significantly $(p<0.001)$ reduced neutrophil migration compared with control NS-CLP mice and induced a marked increase in bacteremia (Figures $1 \mathrm{~B}$ and $1 \mathrm{C}$ ). No bacteria were detected in the sham and sham plus RTX groups (data not shown). Taken together, these results demonstrate a critical role for CXCR2 signaling in the migration of neutrophils to the primary site of infection and, consequently, for survival during sepsis.

\section{Blockade of iNOS Prevents Down-regulation of CXCR2 in Circulating Neutrophils and Impairment of Neutrophil Migration during Severe Sepsis}

We have previously demonstrated that the impairment of neutrophil migration to an infectious focus in animals subjected to severe sepsis is prevented by blockade of iNOS $(8,9)$. To determine whether the loss of CXCR2 might be associated with the enhanced production of NO observed during sepsis, neutrophil migration in the peritoneal cavity and CXCR2 cell surface expression levels in circulating neutrophils were assessed 6 and 2 hours, respectively, after induction of septic peritonitis in control and iNOS inhibitor-treated mice. Consistent with our previous data $(8,9)$, Figure $2 \mathrm{~A}$ shows that inhibition of iNOS by pretreatment of mice subjected to S-CLP with aminoguanidine or L-canavanine prevented the impairment of neutrophil migration into the peritoneal cavity 6 hours after CLP. Similarly, neutrophil migration was restored in $\mathrm{iNOS}^{-/-}$mice subjected to S-CLP (Figure 2A). Interestingly, inhibition of iNOS with aminoguanidine failed to prevent the impairment of neutrophil migration when given after repertaxin treatment (Figure 2A), reinforcing the dominant role of CXCR2 receptor in neutrophil migration.

Flow cytometric analysis showed that 2 hours after surgery CXCR2 expression in circulating neutrophils from S-CLP mice was significantly reduced by 63 and $75 \%$ when compared with NS-CLP and sham-operated mice, respectively (Figure 2B). Interestingly, in experiments in which iNOS was blockaded with aminoguanidine or L-canavanine, and in experiments with iNOS $^{-/}$mice, the observed down-regulation of CXCR2 in the S-CLP group was attenuated (Figure 2B). The expression of CXCR2 on neutrophils of mice subjected to S-CLP and in which iNOS was inhibited pharmacologically or genetically was not significantly different from that observed on neutrophils from the NS-CLP group (Figure 2B).

The reduction of cell surface CXCR2 levels correlates with the endocytosis or internalization of CXCR2 receptors (36). Using fluorescence microscopy, we determined the cell distribution of CXCR2 in circulating neutrophils 2 hours after sepsis induction. As shown in Figure 3, sham-operated mice (Figure 3A) had superficial and homogeneous membrane expression of CXCR2 when compared with animals subjected to CLP. Neutrophils obtained from NS-CLP mice showed an increase in cytoplasmic fluorescence, with the formation of internal clusters of CXCR2 (Figure 3B). However, mice subjected to S-CLP (Figure 3C) had an accentuated loss of neutrophil membrane CXCR2 and a marked increase in the formation of dense fluorescent dot complexes in the cytoplasm. Confocal scanning images revealed that the reduction of neutrophil CXCR2 expression in S-CLP 

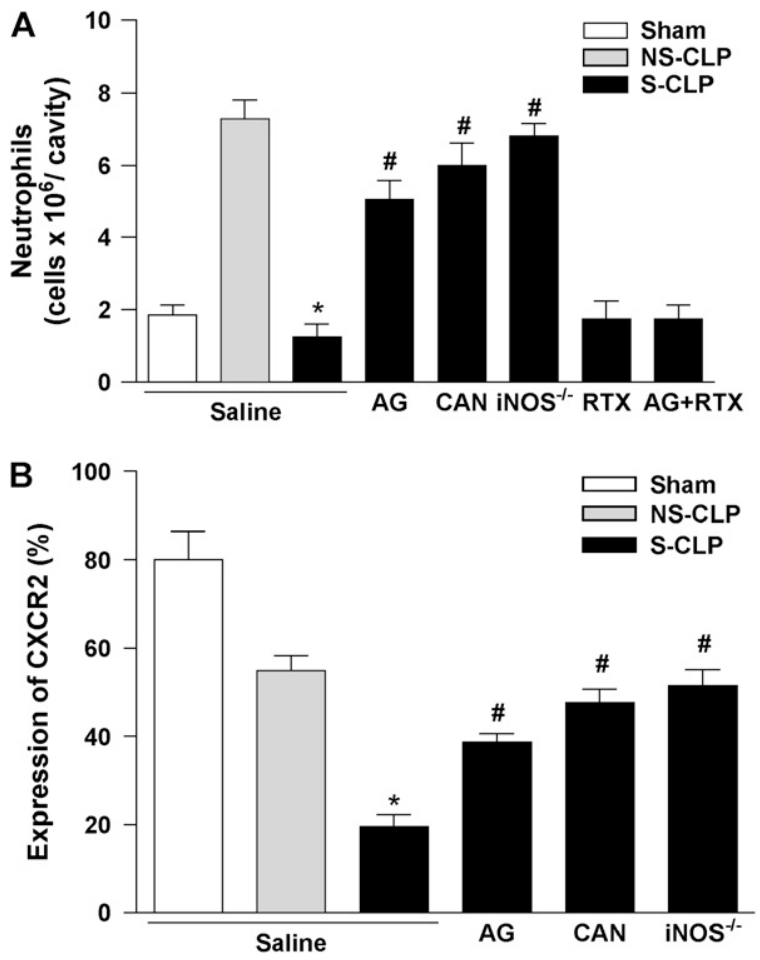

Figure 2. Blockade of inducible nitric oxide synthase (iNOS) prevents neutrophil migration to the peritoneal cavity and attenuates neutrophil CXCR2 down-regulation in severe sepsis. ( $A$ ) Neutrophil migration into the peritoneal cavity of animals pretreated with saline (vehicle), aminoguanidine (AG), L-canavanine (CAN), repertaxin (RTX), or AG plus RTX and subjected to CLP, and of iNOS ${ }^{-1-}$ mice subjected to CLP. Results are represented as means and SEM of the number of neutrophils in the peritoneal cavity $(n=8)$. (B) Purified neutrophils from mice pretreated with saline (vehicle), AG, or CAN, or from iNOS ${ }^{-/}$mice, and subjected to CLP ( $2 \mathrm{~h}$ after surgery) were labeled with fluorescein isothiocyanateconjugated Gr-1 and phycoerythrin-conjugated CXCR2 antibodies and CXCR2 expression was determined by flow cytometry. Data represent means and SEM of three different experiments. In both $(A)$ and $(B):{ }^{*} \mathrm{p}<$ 0.001 compared with sham-operated animals, $\# p<0.01$ compared with S-CLP mice pretreated with saline (analysis of variance, followed by the Bonferroni test).

mice was due to protein internalization (data not shown). Conversely, neutrophils obtained from iNOS ${ }^{--}$mice subjected to S-CLP (Figure 3D) had CXCR2 distribution similar to that observed in the neutrophils of mice subjected to NS-CLP. Taken together, these data show that impairment of neutrophil migration to the infectious focus in severe sepsis is associated with reduction of CXCR2 protein expression on the membrane of circulating neutrophils. The blockade of iNOS prevented internalization.

\section{Reestablishment of CXCR2 Responsiveness by Blockade of iNOS in Severe Sepsis}

In vitro studies have demonstrated that CXCR2 down-regulation may induce a reduction in neutrophil response to chemokines that bind to CXCR2 (37). A series of in vitro and in vivo experiments were conducted in an attempt to investigate the integrity of CXCR2 function during sepsis. First, we examined in the mesenteric postcapillary venules the leukocyte-endothelium interaction (adhesion and migration) in response to stimulation with MIP-2, a CXC-ELR ${ }^{+}$chemokine that binds CXCR2. Figure 4 shows that application of MIP-2 over the mesentery of sham- operated or mice subjected to NS-CLP increased neutrophil adhesion and migration in a similar manner. However, there was a decrease in neutrophil adhesion and migration in S-CLP mice given MIP-2 (Figure 4). Pretreatment with aminoguanidine or L-canavanine restored leukocyte adhesion and mesenterium transmigration in response to MIP-2 in S-CLP animals. Similar results were observed in NOS $^{-/}$mice subjected to S-CLP (data not shown). The reduction of CXCR2 expression and of neutrophil adhesion and migration observed in S-CLP mice was not a consequence of a decrease in the number of circulating neutrophils, as the number of neutrophils were not different in the groups 2 hours after surgery (data not shown).

Changes in intracellular calcium $\left(\mathrm{Ca}^{2+}\right)$ concentration are classically associated with chemokine activation of cells and provide a mechanism by which receptor engagement and response specificity may be examined (38). Neutrophils from mice subjected to S-CLP treated or not treated with iNOS inhibitors were collected 2 hours after surgery and stimulated in vitro with MIP-2 (20 ng/ml). $\mathrm{Ca}^{2+}$ mobilization in neutrophils from S-CLP mice treated with aminoguanidine or L-canavanine had an increased response to MIP-2 compared with control S-CLP mice (7.5 \pm 0.5 and $10.1 \pm 0.3$ vs. $2.8 \pm 0.5$ relative fluorescence; $\mathrm{n}=5$, $\mathrm{p}<0.05$ by analysis of variance and Bonferroni $t$ test), suggesting a recovery of CXCR2 mediating the intracellular calcium mobilization response.

\section{Nitric Oxide Down-modulates CXCR2, But Not CXCR1, Expression in Human Neutrophils}

To assess whether the preceding observations applied to human neutrophils, the chemotaxis of blood neutrophils in response to CXCL8 (10 ng/ml) was studied after pretreatment of cells with an NO donor (SNAP, $300 \mu \mathrm{M})$, citrulline $(30 \mu \mathrm{M})$, CXCL8 (30 ng/ml), or SNAP plus CXCL8. As shown in Figure 5, SNAP pretreatment resulted in a marked inhibition of CXCL8-induced chemotaxis. Pretreatment with citrulline had no effect on chemotaxis at the concentration and time studied. Consistent with its ability to desensitize neutrophils (39), pretreatment with CXCL8 induced a marked inhibitory effect on the chemotactic response to CXCL8. The latter effect was not enhanced when coincubated with SNAP. Incubation with aminoguanidine before the first addition of CXCL8 was used to determine whether the inhibitory effect of CXCL8 on CXCL8-induced chemotaxis was NO dependent. The blockade of iNOS partially prevented the inhibitory effect of CXCL8 (Figure 5).

To confirm the critical role of nitric oxide in chemokine receptor down-regulation, we examined the effect of NO donor exposure on the expression of CXCR1 and CXCR2 on human neutrophils and of CXCR2 on murine neutrophils. Flow cytometric analysis showed that pretreatment with the NO donor (SNAP) failed to affect the expression of CXCR1 (Figures 6A and 6D), but significantly decreased the expression of CXCR2 on human neutrophils (Figures 6B and 6E). SNAP also significantly decreased CXCR2 expression on murine neutrophils (Figure 6C). In contrast, pretreatment with citrulline had no effect on CXCR1 and CXCR2 expression on murine or human neutrophils (Figure 6). Together, these data provide direct evidence that NO downregulates CXCR2, but not CXCR1, expression on neutrophils. Finally, we show that CXCR2, but not CXCR1, expression is regulated by an agonist-dependent mechanism. Human neutrophils pretreated with CXCL8 (human CXCR1 and CXCR2 ligand) or murine neutrophils pretreated with KC or MIP-2 (murine CXCR2 ligands), displayed a significant decrease in cell surface CXCR2 expression (Figures 6B and 6C). 
A Sham

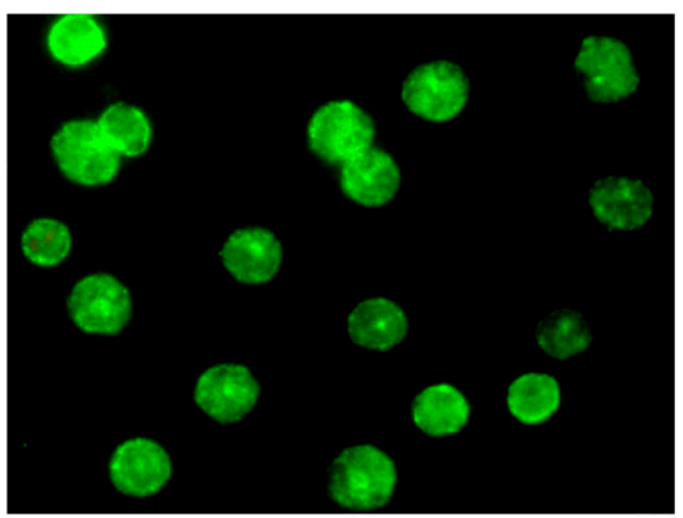

C S-CLP

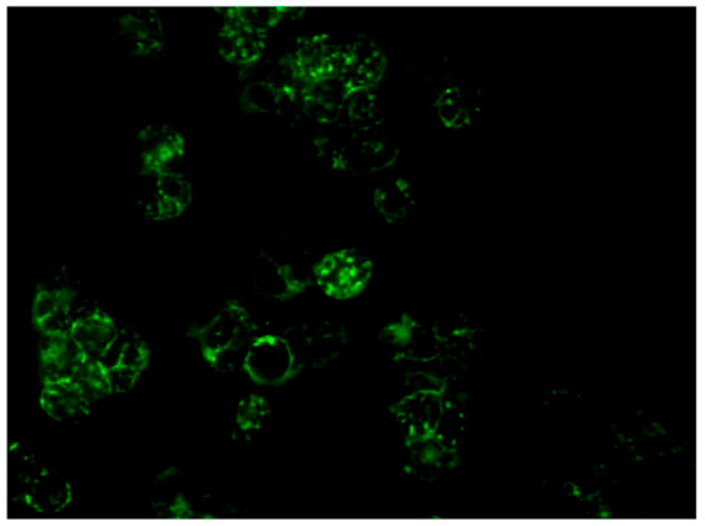

B NS-CLP

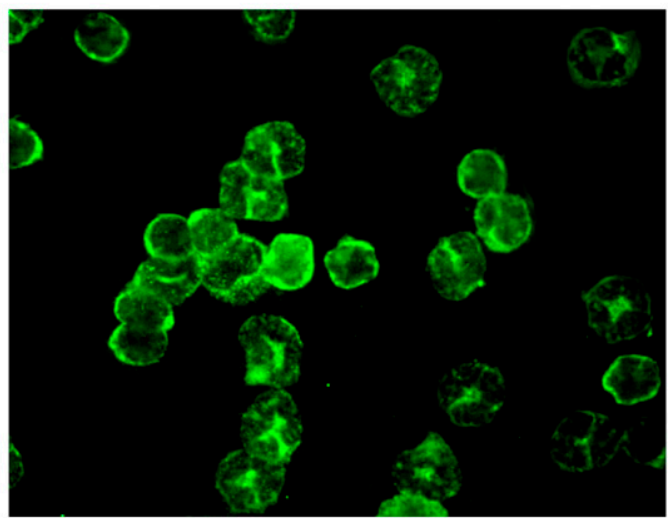

D S-CLP (iNOS ${ }^{-1}$ )

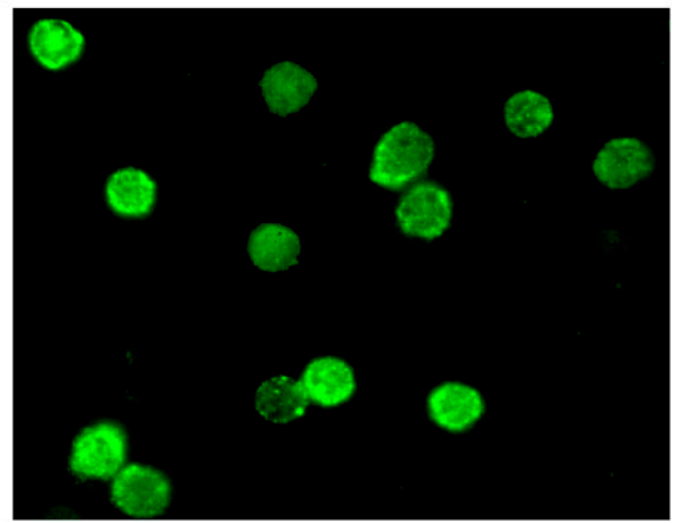

Figure 3. Down-regulation of neutrophil CXCR2 in severe sepsis was attenuated by iNOS blockade. Wild-type mice (C57BL/6) subjected to sham surgery, NS-CLP, or S-CLP and iNOS $^{-1-}$ mice subjected to S-CLP were killed 2 hours after surgery and purified neutrophils were labeled with anti-CXCR2 antibody conjugated with secondary anti-lgG antibody labeled with Alexa-Fluor 488. (Original magnification: $\times 1,000$.)

\section{DISCUSSION}

Neutrophil recruitment to the site of infection is an essential step for the control of invading pathogens (1-3). CXC-ELR ${ }^{+}$ chemokines display a central role in mediating the signaling cascade that targets neutrophils to sites of infection by binding to receptors on the surface of these cells (40). Thus, downregulation of chemokine receptors and/or chemokine unresponsiveness may be critical to the impairment of neutrophil recruitment during infection. In this respect, there is evidence to suggest that human and murine chemokine receptors are down-regulated in neutrophils during severe sepsis. It has been previously demonstrated that the expression of CXCR2, but not CXCR1, is down-regulated on the membrane of neutrophils obtained from septic patients $(28,31)$. Furthermore, we have demonstrated that neutrophils obtained from patients with severe sepsis display a marked reduction of CXCL8-induced chemotaxis (41). The mechanisms underlying the loss of chemokine receptors from the neutrophil surface have not been explored. In the present study, we sought to investigate the involvement of CXCR2 in neutrophil migration during sepsis. Our results showed that there was clear down-regulation of this receptor that correlated with the failure of neutrophils to migrate to the site of infection. We then evaluated the role of $\mathrm{NO}$ in mediating the down-regulation of CXCR2 expression on neutrophils and in CXCR2-dependent neutrophil recruitment to a site of infection during severe sepsis.

Initial experiments investigated the effect of CXCR2 on the outcome of sepsis in mice. We showed that inhibition of CXCR2 chemokine receptor by repertaxin, a noncompetitive allosteric blocker of murine CXCR2 (33), resulted in marked reduction of neutrophil migration to the infectious focus during nonsevere sepsis. Failure of neutrophils to migrate was associated with failure of the host to deal with the infectious insult and, conse- quently, with mortality rates similarly to those observed in mice subjected to severe sepsis. Accordingly, one study has shown that CXCR2 down-regulation may predispose to pneumonia after trauma or other inflammatory conditions that lead to the systemic inflammatory response syndrome (29). Altogether, these data provide evidence suggesting that CXCR2 signaling is critical to regulate the migration of neutrophils and host defense during bacterial infection.

Previous studies from our laboratory have shown that marked impairment of neutrophil migration to the infectious focus is observed in severe sepsis, resulting in the inability of the host to restrict the local infection. These studies have shown that the loss of local control of infection results in dissemination of bacteria and high mortality rates in animal models $(6,8,9,11)$. More importantly, we have previously shown that neutrophils obtained from septic patients had reduced chemotaxis to CXCL8 and that the loss of response correlated well with the severity of disease (13). In animals, NO seems to be an important mediator of the impairment of neutrophil migration in sepsis, as the failure to migrate is prevented by pharmacologic or genetic blockade of iNOS (7-9). Taking into account this evidence, and the findings that CXCR2 is down-regulated in severe sepsis (28, 31 ), we postulated that CXCR2 down-regulation might be responsible for the impairment of neutrophil migration observed in severe sepsis and that iNOS-derived NO mediates the receptor down-regulation. We observed both impairment of the migration of neutrophils to the infection focus and down-regulation of the expression of CXCR2 on circulating neutrophils in S-CLP mice. Data obtained by flow cytometry, immunofluorescence, and scanning confocal microscopy clearly indicated that neutrophils from S-CLP mice showed a decrease in membrane CXCR2 localization and increased internalization of $\mathrm{CXCR} 2$, when compared 

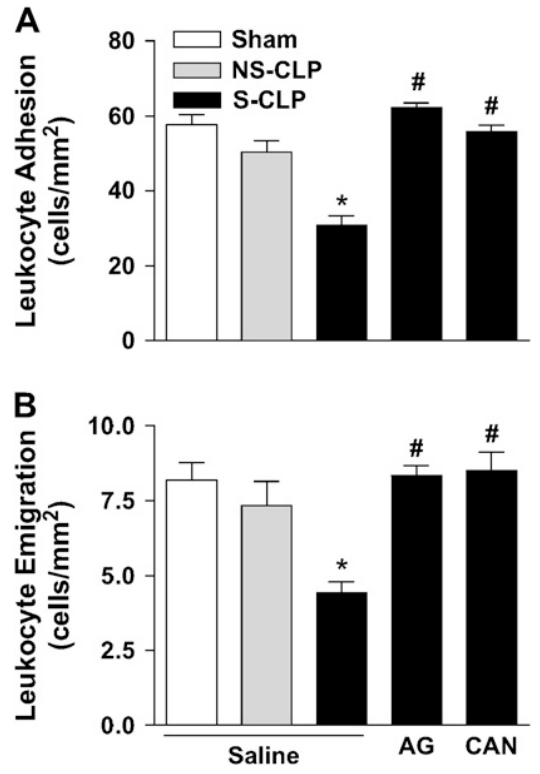

Figure 4. iNOS blockade prevents neutrophil desensitization to macrophage inflammatory protein (MIP)-2 in severe sepsis. Adhesion $(A)$ and migration $(B)$ of leukocytes in MIP-2-stimulated mesenteric venules of sham-operated mice or mice subjected to NS-CLP or S-CLP pretreated with saline (vehicle), aminoguanidine (AG), or L-canavanine (CAN). Similar results were observed in the vehicle-treated sham group and sham groups treated with iNOS inhibitors (CAN and AG). Measurements were evaluated for 10 minutes, 2 hours after surgery. The leukocyteendothelium interaction was stimulated by local administration of MIP-2 $(20 \mathrm{ng} / \mathrm{ml})$ into the mesenterium $(\mathrm{n}=8)$. ${ }^{*} \mathrm{p}<0.01$ compared with NS-CLP group; $" \mathrm{p}<0.01$ compared with S-CLP mice pretreated with saline (analysis of variance, followed by Bonferroni test).

with NS-CLP mice. Both pharmacologic and genetic inhibition of iNOS attenuated CXCR2 internalization, and prevented the impairment of neutrophil migration in S-CLP mice. The latter results support our hypothesis that NO derived from iNOS mediates the down-regulation of the chemokine receptor during se-

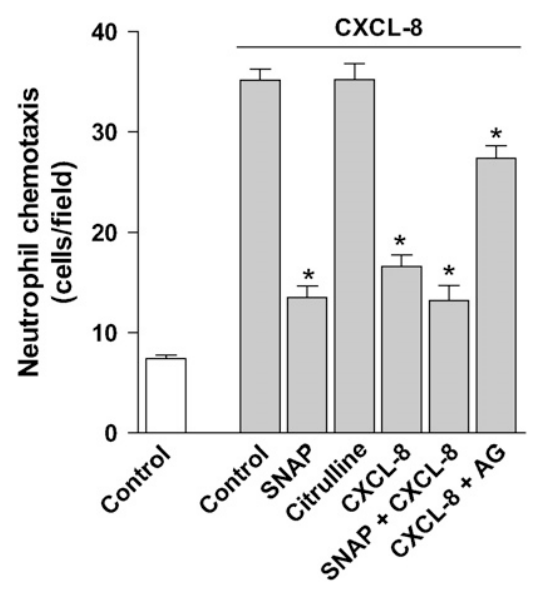

Figure 5. Nitric oxide inhibits human neutrophil chemotaxis. Human purified blood neutrophils were preincubated with medium (Control), NO donor (S-nitroso- $N$-acetyl-D,L-penicillamine [SNAP], $300 \mu \mathrm{M}$ ), citrulline $(30 \mu \mathrm{M}), \mathrm{CXCL8}(30 \mathrm{ng} / \mathrm{ml}), \mathrm{CXCL8}$ plus SNAP, or iNOS inhibitor (AG, $50 \mu \mathrm{M}$ ) plus $\mathrm{CXCL8}$ for 2 hours at $37^{\circ} \mathrm{C}$ before chemotaxis to CXCL8 $(10 \mathrm{ng} / \mathrm{ml})$ in Boyden chamber assays. Experiments were performed three times, each in triplicate. Results are expressed as means and SEM. ${ }^{*} p<0.01$ compared with control in CXCL8. vere sepsis. To gain further insight concerning the significance of iNOS blockade in CXCR2 internalization dynamics, we examined the leukocyte-endothelium interaction (adhesion and emigration) in response to MIP-2 (CXCR2 ligand) stimulation in mesenteric postcapillary venules of S-CLP mice. It was observed that MIP-2 induced lower adhesion and transmigration in S-CLP mice, compared with those induced in NS-CLP mice. In contrast, blockade of iNOS reestablished the leukocyteendothelium interactions. Inhibition of NO production by treatment of S-CLP mice with an iNOS inhibitor failed to reestablish neutrophil migration when the iNOS inhibitor was given in association with a CXCR2 blocker. The latter results reinforce the concept that iNOS-derived NO accounts for the down-regulation of CXCR2. Moreover, the results suggest that CXCR2 downregulation is a key event for the impairment of the migration of neutrophils during severe sepsis.

There is evidence that severe infection is associated with a systemic mobilization of neutrophils from the bone marrow and that these newly released neutrophils preferentially sequester in lung and are slow to migrate into infected tissues (42). In this context, there is evidence that heterogeneity in the maturation of circulating neutrophils accounts for the variability in cytoskeletal rearrangement and migration in chemokine-induced signaling (43). In the present study, the circulating neutrophil counts are similar in both vehicle- and iNOS inhibitor-treated groups 2 hours after S-CLP, suggesting that, under our experimental conditions, the reduction of neutrophil migration is not a consequence of alteration in neutrophil bone marrow mobilization.

It is well demonstrated that desensitization of chemokine receptors is induced by continuous contact with the respective ligands (44). The ligand-receptor complex is internalized into vesicles through dynamin-mediated formation of clathrin-coated pits and thereafter the receptors are rapidly transported to early and recycling endosomal compartments (21). Taking these findings into account, we confirmed that NO mediates CXCR2 desensitization in vitro. It was observed that preincubation of human neutrophils with CXCL8 resulted in reduction of the chemotactic response to CXCL8 and that the process was inhibited by iNOS blockade. Moreover, neutrophils preincubated with NO donors displayed a marked reduction in chemotactic response to CXCL8. As mentioned previously, in humans $\mathrm{CXC} \mathrm{ELR}^{+}$chemokines act through two receptors, designated CXCR1 and CXCR2 $(17,18)$, whereas in mice they act through CXCR2 (19). There was a significant reduction of CXCR2, but not CXCR1, expression on human neutrophils and of CXCR2 on murine neutrophils after NO donor incubation. Moreover, human neutrophils pretreated with CXCL8 (a ligand for human CXCR1 and CXCR2) or murine neutrophils pretreated with KC or MIP-2 (ligands for the murine CXCR2) displayed a significant decrease in cell surface CXCR2 expression. Taken together, these observations provide direct evidence that NO down-regulates CXCR2 expression, which in turn leads to a reduction of the chemotactic response to the CXCR2-active ligand.

In agreement with our data, several studies in the literature indicate that NO donors or induction of iNOS promotes downregulation of various GPCRs, including $\beta$-adrenoreceptors in myocytes (45-47), angiotensin II receptors in vascular smooth muscle cells (48), and muscarinic receptors in Chinese hamster ovary cell lines (49). The mechanisms by which NO induces CXCR2 down-regulation were not investigated here. One interesting possibility is that NO may react with superoxide-forming peroxynitrite, which may account for nitration of essential cysteine and/or tyrosine residues of GPCRs $(50,51)$. Clearly, further experiments are necessary to dissect the mechanism underlying NO-mediated CXCR2 down-regulation during severe sepsis. 

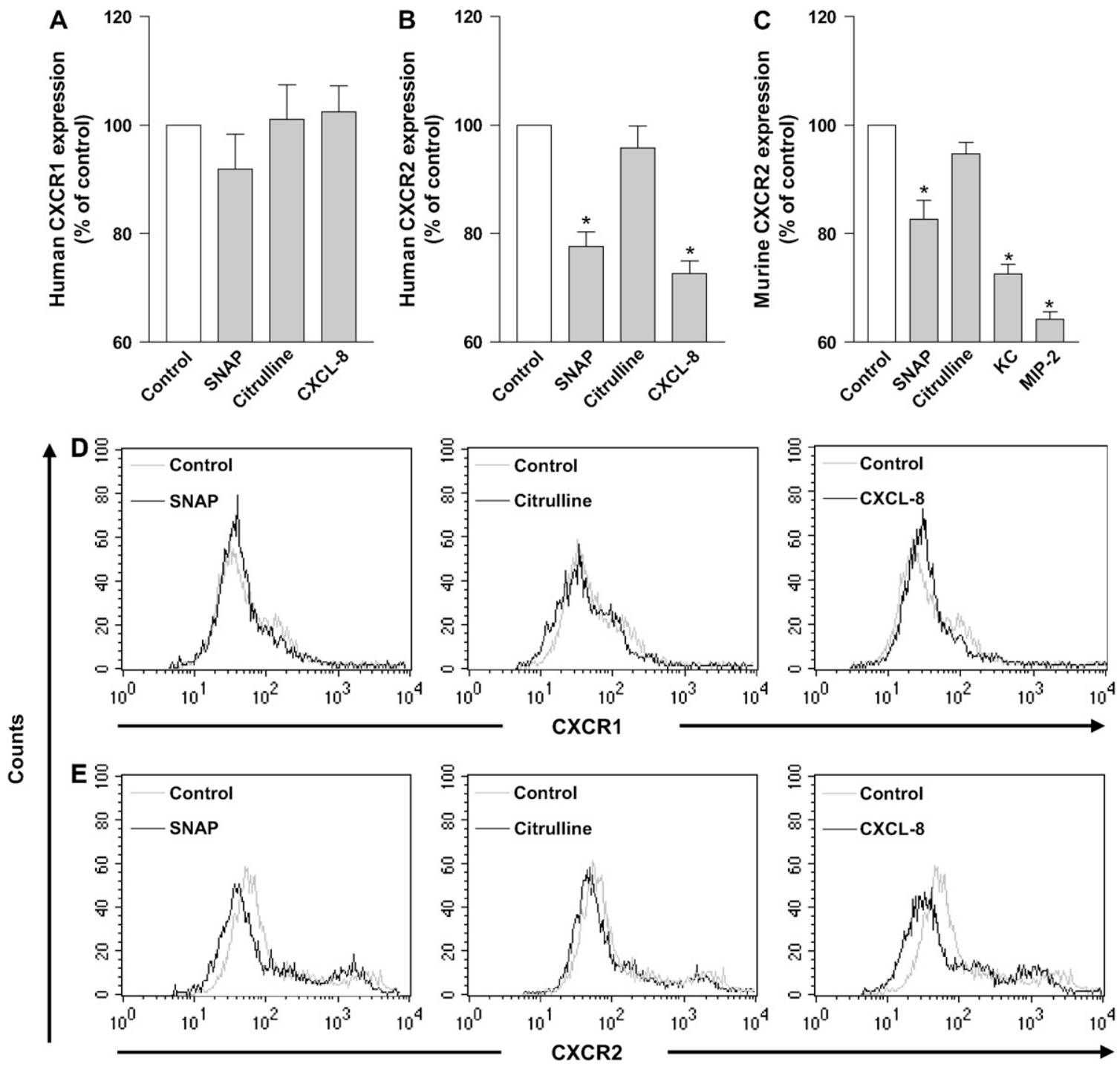

Figure 6. Nitric oxide directly down-modulates CXCR2 expression on murine and human neutrophils. Purified blood neutrophils were preincubated with RPMI (Control), NO donor (SNAP, $300 \mu \mathrm{M})$, citrulline $(30 \mu \mathrm{M}), \mathrm{CXCL8}(30 \mathrm{ng} / \mathrm{ml}), \mathrm{KC}(30 \mathrm{ng} / \mathrm{ml})$, or MIP-2 (30 ng/ml) for $2 \mathrm{hours}$ at $37^{\circ} \mathrm{C}$, and human CXCR1 $(A)$, human CXCR2 $(B)$, and murine CXCR2 $(C)$ expression was measured by flow cytometry as described in METHODs. (D and E) Illustrative histogram for human CXCR1 and CXCR2, respectively. Data are presented as means and SEM of percent specific change from control (neutrophils stimulated with medium) after subtraction of nonspecific binding of isotype-matched controls. Experiments were performed three times, each in triplicate. ${ }^{*} \mathrm{p}<0.05$ compared with control.

In conclusion, the failure of neutrophils to migrate to an infection focus during severe sepsis in mice is associated with excessive NO production and NO-dependent regulation of the expression of CXCR2 on the neutrophil surface. These effects of $\mathrm{NO}$ production on CXCR2 expression may explain the ability of excess $\mathrm{NO}$ to prevent the migration of neutrophils to the focus of infection during severe sepsis.

Conflict of Interest Statement: None of the authors has a financial relationship with a commercial entity that has an interest in the subject of this manuscript.

Acknowledgment: The authors thank Giuliana Bertozi, Fabíola Leslie Mestriner, and Ana Kátia dos Santos for technical assistance.

\section{References}

1. Wagner JG, Roth RA. Neutrophil migration during endotoxemia. J Leukoc Biol 1999;66:10-24.

2. Niggli V. Signaling to migration in neutrophils: importance of localized pathways. Int J Biochem Cell Biol 2003;35:1619-1638.
3. Simon SI, Green CE. Molecular mechanics and dynamics of leukocyte recruitment during inflammation. Annu Rev Biomed Eng 2005;7:151185.

4. Alves-Filho JC, Tavares-Murta BM, Barja-Fidalgo C, Benjamim CF, Basile-Filho A, Arraes SM, Cunha FQ. Neutrophil function in severe sepsis. Endocr Metab Immune Disord Drug Targets 2006;6:151-158.

5. Alves-Filho JC, Benjamim C, Tavares-Murta BM, Cunha FQ. Failure of neutrophil migration toward infectious focus in severe sepsis: a critical event for the outcome of this syndrome. Mem Inst Oswaldo Cruz 2005;100:223-226.

6. Alves-Filho JC, de Freitas A, Russo M, Cunha FQ. Toll-like receptor 4 signaling leads to neutrophil migration impairment in polymicrobial sepsis. Crit Care Med 2006;34:461-470.

7. Benjamim CF, Silva JS, Fortes ZB, Oliveira MA, Ferreira SH, Cunha FQ. Inhibition of leukocyte rolling by nitric oxide during sepsis leads to reduced migration of active microbicidal neutrophils. Infect Immun 2002;70:3602-3610.

8. Benjamim CF, Ferreira SH, Cunha FQ. Role of nitric oxide in the failure of neutrophil migration in sepsis. J Infect Dis 2000;182:214-223.

9. Crosara-Alberto DP, Darini AL, Inoue RY, Silva JS, Ferreira SH, Cunha FQ. Involvement of $\mathrm{NO}$ in the failure of neutrophil migration in sepsis induced by Staphylococcus aureus. Br J Pharmacol 2002;136:645-658. 
10. Tavares-Murta BM, Machado JS, Ferreira SH, Cunha FQ. Nitric oxide mediates the inhibition of neutrophil migration induced by systemic administration of LPS. Inflammation 2001;25:247-253.

11. Rios-Santos F, Benjamim CF, Zavery D, Ferreira SH, Cunha FQ. A critical role of leukotriene $\mathrm{B}_{4}$ in neutrophil migration to infectious focus in cecal ligation and puncture sepsis. Shock 2003;19:61-65.

12. Casey LC, Balk RA, Bone RC. Plasma cytokine and endotoxin levels correlate with survival in patients with the sepsis syndrome. Ann Intern Med 1993;119:771-778.

13. Tavares-Murta BM, Zaparoli M, Ferreira RB, Silva-Vergara ML, Oliveira $\mathrm{CH}$, Murta EF, Ferreira SH, Cunha FQ. Failure of neutrophil chemotactic function in septic patients. Crit Care Med 2002;30:1056-1061.

14. Cunha FQ, Moss DW, Leal LM, Moncada S, Liew FY. Induction of macrophage parasiticidal activity by Staphylococcus aureus and exotoxins through the nitric oxide synthesis pathway. Immunology 1993; 78:563-567.

15. Ghaffari A, Miller CC, McMullin B, Ghahary A. Potential application of gaseous nitric oxide as a topical antimicrobial agent. Nitric Oxide 2005;14:21-29.

16. Broaddus VC, Boylan AM, Hoeffel JM, Kim KJ, Sadick M, Chuntharapai A, Hebert CA. Neutralization of IL-8 inhibits neutrophil influx in a rabbit model of endotoxin-induced pleurisy. J Immunol 1994;152: 2960-2967.

17. Lee J, Horuk R, Rice GC, Bennett GL, Camerato T, Wood WI. Characterization of two high affinity human interleukin-8 receptors. J Biol Chem 1992;267:16283-16287.

18. Ahuja SK, Murphy PM. The CXC chemokines growth-regulated oncogene (GRO) $\alpha$, GRO $\beta$, GRO $\gamma$, neutrophil-activating peptide-2, and epithelial cell-derived neutrophil-activating peptide-78 are potent agonists for the type B, but not the type A, human interleukin- 8 receptor. J Biol Chem 1996;271:20545-20550.

19. Bozic CR, Gerard NP, von Uexkull-Guldenband C, Kolakowski LF Jr, Conklyn MJ, Breslow R, Showell HJ, Gerard C. The murine interleukin 8 type $\mathrm{B}$ receptor homologue and its ligands: expression and biological characterization. J Biol Chem 1994;269:29355-29358.

20. Jones SA, Moser B, Thelen M. A comparison of post-receptor signal transduction events in Jurkat cells transfected with either IL-8R1 or IL-8R2: chemokine mediated activation of p42/p44 MAP-kinase (ERK-2). FEBS Lett 1995;364:211-214.

21. Chuntharapai A, Kim KJ. Regulation of the expression of IL-8 receptor A/B by IL-8: possible functions of each receptor. J Immunol 1995;155: 2587-2594.

22. Tateda K, Moore TA, Newstead MW, Tsai WC, Zeng X, Deng JC, Chen G, Reddy R, Yamaguchi K, Standiford TJ. Chemokine-dependent neutrophil recruitment in a murine model of Legionella pneumonia: potential role of neutrophils as immunoregulatory cells. Infect Immun 2001;69:2017-2024.

23. Mehrad B, Strieter RM, Moore TA, Tsai WC, Lira SA, Standiford TJ. CXC chemokine receptor-2 ligands are necessary components of neutrophil-mediated host defense in invasive pulmonary aspergillosis. J Immunol 1999;163:6086-6094.

24. Tsai WC, Strieter RM, Mehrad B, Newstead MW, Zeng X, Standiford TJ. CXC chemokine receptor CXCR2 is essential for protective innate host response in murine Pseudomonas aeruginosa pneumonia. Infect Immun 2000;68:4289-4296.

25. Moore TA, Newstead MW, Strieter RM, Mehrad B, Beaman BL, Standiford TJ. Bacterial clearance and survival are dependent on CXC chemokine receptor-2 ligands in a murine model of pulmonary Nocardia asteroides infection. J Immunol 2000;164:908-915.

26. Prado GN, Suzuki H, Wilkinson N, Cousins B, Navarro J. Role of the $\mathrm{C}$ terminus of the interleukin 8 receptor in signal transduction and internalization. J Biol Chem 1996;271:19186-19190.

27. Sabroe I, Williams TJ, Hebert CA, Collins PD. Chemoattractant crossdesensitization of the human neutrophil IL-8 receptor involves receptor internalization and differential receptor subtype regulation. $J$ Immunol 1997;158:1361-1369.

28. Cummings CJ, Martin TR, Frevert CW, Quan JM, Wong VA, Mongovin SM, Hagen TR, Steinberg KP, Goodman RB. Expression and function of the chemokine receptors CXCR1 and CXCR2 in sepsis. J Immunol 1999;162:2341-2346.

29. Tarlowe MH, Duffy A, Kannan KB, Itagaki K, Lavery RF, Livingston DH, Bankey P, Hauser CJ. Prospective study of neutrophil chemokine responses in trauma patients at risk for pneumonia. Am J Respir Crit Care Med 2005;171:753-759.

30. Quaid GA, Cave C, Robinson C, Williams MA, Solomkin JS. Preferential loss of CXCR-2 receptor expression and function in patients who have undergone trauma. Arch Surg 1999;134:1367-1371 (discussion 1371-1372)

31. Chishti AD, Shenton BK, Kirby JA, Baudouin SV. Neutrophil chemotaxis and receptor expression in clinical septic shock. Intensive Care Med 2004:30:605-611.

32. Wichterman KA, Baue AE, Chaudry IH. Sepsis and septic shock: a review of laboratory models and a proposal. J Surg Res 1980;29:189-201.

33. Bertini R, Allegretti M, Bizzarri C, Moriconi A, Locati M, Zampella G, Cervellera MN, Di Cioccio V, Cesta MC, Galliera E, et al. Noncompetitive allosteric inhibitors of the inflammatory chemokine receptors CXCR1 and CXCR2: prevention of reperfusion injury. Proc Natl Acad Sci USA 2004;101:11791-11796.

34. Cotter MJ, Norman KE, Hellewell PG, Ridger VC. A novel method for isolation of neutrophils from murine blood using negative immunomagnetic separation. Am J Pathol 2001;159:473-481.

35. Fraticelli A, Serrano CV Jr, Bochner BS, Capogrossi MC, Zweier JL. Hydrogen peroxide and superoxide modulate leukocyte adhesion molecule expression and leukocyte endothelial adhesion. Biochim Biophys Acta 1996;1310:251-259.

36. Rose JJ, Foley JF, Murphy PM, Venkatesan S. On the mechanism and significance of ligand-induced internalization of human neutrophil chemokine receptors CXCR1 and CXCR2. J Biol Chem 2004;279:2437224386.

37. Adams JM, Hauser CJ, Livingston DH, Lavery RF, Fekete Z, Deitch EA. Early trauma polymorphonuclear neutrophil responses to chemokines are associated with development of sepsis, pneumonia, and organ failure. J Trauma 2001;51:452-456. (Discussion 456-457.)

38. Murphy PM. The molecular biology of leukocyte chemoattractant receptors. Annu Rev Immunol 1994;12:593-633.

39. Ben-Baruch A, Grimm M, Bengali K, Evans GA, Chertov O, Wang JM, Howard OM, Mukaida N, Matsushima K, Oppenheim JJ. The differential ability of IL-8 and neutrophil-activating peptide- 2 to induce attenuation of chemotaxis is mediated by their divergent capabilities to phosphorylate CXCR2 (IL-8 receptor B). J Immunol 1997;158: 5927-5933.

40. Yoshie O, Imai T, Nomiyama H. Chemokines in immunity. Adv Immunol 2001;78:57-110

41. Arraes SM, Freitas MS, da Silva SV, de Paula Neto HA, Alves-Filho JC, Martins MA, Basile-Filho A, Tavares-Murta BM, Barja-Fidalgo C, Cunha FQ. Impaired neutrophil chemotaxis in sepsis associates with GRK expression and inhibition of actin assembly and tyrosine phosphorylation. Blood 2006;108:2906-2913.

42. Sato Y, Van Eeden SF, English D, Hogg JC. Pulmonary sequestration of polymorphonuclear leukocytes released from bone marrow in bacteremic infection. Am J Physiol 1998;275:L255-L261.

43. Tanji-Matsuba K, van Eeden SF, Saito Y, Okazawa M, Klut ME, Hayashi $\mathrm{S}$, Hogg JC. Functional changes in aging polymorphonuclear leukocytes. Circulation 1998;97:91-98.

44. Khandaker MH, Mitchell G, Xu L, Andrews JD, Singh R, Leung H, Madrenas J, Ferguson SS, Feldman RD, Kelvin DJ. Metalloproteinases are involved in lipopolysaccharide- and tumor necrosis factor$\alpha$-mediated regulation of CXCR1 and CXCR2 chemokine receptor expression. Blood 1999;93:2173-2185.

45. Ziolo MT, Katoh H, Bers DM. Expression of inducible nitric oxide synthase depresses $\beta$-adrenergic-stimulated calcium release from the sarcoplasmic reticulum in intact ventricular myocytes. Circulation 2001;104:2961-2966.

46. Stojanovic MO, Ziolo MT, Wahler GM, Wolska BM. Anti-adrenergic effects of nitric oxide donor SIN-1 in rat cardiac myocytes. Am J Physiol Cell Physiol 2001;281:C342-C349.

47. Ziolo MT, Maier LS, Piacentino V III, Bossuyt J, Houser SR, Bers DM. Myocyte nitric oxide synthase 2 contributes to blunted $\beta$-adrenergic response in failing human hearts by decreasing $\mathrm{Ca}^{2+}$ transients. Circulation 2004;109:1886-1891.

48. Cahill PA, Redmond EM, Foster C, Sitzmann JV. Nitric oxide regulates angiotensin II receptors in vascular smooth muscle cells. Eur J Pharmacol 1995;288:219-229.

49. Maggio R, Barbier P, Toso A, Barletta D, Corsini GU. Sodium nitroprusside induces internalization of muscarinic receptors stably expressed in Chinese hamster ovary cell lines. J Neurochem 1995;65:943-946.

50. Lewis SJ, Hoque A, Walton TM, Kooy NW. Potential role of nitration and oxidation reactions in the effects of peroxynitrite on the function of $\beta$-adrenoceptor sub-types in the rat. Eur J Pharmacol 2005;518:187194.

51. Giuntini J, Giusti L, Lucacchini A, Mazzoni MR. Modulation of A1 adenosine receptor signaling by peroxynitrite. Biochem Pharmacol 2004;67:375-383. 